SINAI Journal of Applied Sciences

\title{
FOLIAR SPRAY OF PEA PLANTS WITH MODIFIED EGG ALBUMIN FOR ENHANCING GROWTH AND PRODUCTIVITY
}

\author{
Hany E.M. Ismail ${ }^{1^{*}}$, A. Osman ${ }^{2}$ and M.Z. Sitohy ${ }^{2}$ \\ 1. Dept. Hort., Fac. Agric., Zagazig Univ., Egypt. \\ 2. Dept. Biochem., Fac. Agric., Zagazig Univ., Egypt.
}

\begin{abstract}
This work was carried out during the two successive winter seasons of 2015/2016 and 2016/2017 under sandy soil conditions using drip irrigation system at El-Khattara Experimental Farm, Faculty of Agriculture, Zagazig University, Sharkia Governorate, Egypt, to study the effect of different native and modified egg white protein and total amino acids concentrations as foliar applications on growth, antioxidants, yield and seeds chemical constituent of pea cv. Master B. Foliar spraying of pea plants with native egg albumin (NEA) at $2 \mathrm{ml} / 1$ increased stem length, number of branches/plant and total fresh weight/ plant in both seasons. Foliar spray with hydrolyzed methylated egg albumin (HMEA) at $2 \mathrm{ml} / 1$ increased the concentration of chlorophyll $\mathrm{a}, \mathrm{b}$, and $(\mathrm{a}+\mathrm{b})$ and carotenoids compared to other treatments. Multi amino acids 24\% (MAA) at $2 \mathrm{ml} / 1$ increased $\mathrm{N}$ and total protein, MAA at $1 \mathrm{ml} / 1$ increased $\mathrm{P}(\%)$ and HMEA at $2 \mathrm{ml} / 1$ increased $\mathrm{K}(\%)$ in seeds in both seasons. NEA at $1.5 \mathrm{ml} / 1$ or hydrolyzed native egg albumin (HNEA) at $1 \mathrm{ml} / 1$ increased number of green pod/plant, average green pod weight, yield of green pods/ plant and total yield/ green pod, whereas HMEA at $1.5 \mathrm{ml} / 1$ or MAA at $1.5 \mathrm{ml} 1$ increased number of seeds/ pod.
\end{abstract}

Key words: Peas, egg white protein, methylation, protein hydrolysates, yield.

\section{INTRODUCTION}

Peas (Pisum sativum L.) which belongs to the family Fabaceae is considered the most widely distributed pules in most countries over all the world. It is an excellent source of protein, complex carbohydrates, vitamins (A, B and C), phosphorus, potassium, magnesium, calcium and many other minerals, great nutritional importance due to their high contents of protein, complex carbohydrates, dietary fiber, minerals, vitamins, and antioxidant compounds (Biesaga-Koscielniak et al., 2014). Egg white is a normal source of proteins of famed nutritional and biological advantages (Pihlanto and Korhonen, 2003). Enzymatic hydrolysis is a particular technique to transform native protein to protein (Abdel-Hamid et al., 2016; Osman et al., 2016). Protein hydrolysates are mix of polypeptides, oligopeptides and amino acids (Poullain et al., 1989). (Kauffman $\boldsymbol{e t}$ al., 2007) classified organic bio stimulant compounds, into three major groups on the basis of their source and content: humic substances, seaweed extracts, and amino acids containing products. The last group consists of free amino acids and polypeptides obtained through chemical and/or enzymatic hydrolysis of agro industrial by-products from animal or plant sources or from dedicated biomass crops (Colla et al., 2014).

Recently, there is a growing apprehension on the utilize of animal-derived protein hydrolysates in terms of food safety as

\footnotetext{
* Corresponding author: Tel.: +201005133426
}

E-mail address: hanyeldewah7@gmail.com 
elucidated by the ban of animal-derived protein hydrolysate application on the eaten parts of crops in organic cultivation, Ertani et al. (2009) and Gurav and Jadhav (2013) reported beneficial effects for foliar protein hydrolysates applications on growth, yield and fruit quality of agricultural crops. Protein hydrolysate could also work as plant growth regulators due to the appearance of peptides. Several bioactive peptides generated in a species of plants have been found to have plant hormoneslike activities (Ito et al., 2006; Kondo et al., 2006). Ertani et al. (2009) spotted that uses of plant-derived protein hydrolysates on corn and tomato plants increased value nutrient uptake in particelular nitrogen and iron as a result of increased glutamine synthetase (GS) and nitrate reductase (NR) activities, and $\mathrm{Fe}$ (III)-chelate reductase activity, respectively. Two protein hydrolysate based on fertilizers (PHFs) increased leaf growth and root and motivated morphological changes in root architecture. Besides, the treatments increased NR and GS activities, indicating a positive role of the two hydrolysates in the inducement of nitrate transformation into organic nitrogen (Ertani et al., 2009). The root, shoot dry weight, root area, and root length were significantly higher by 35,21 , 26 , and $24 \%$, respectively, in tomato treated plants with the protein hydrolysate at $6 \mathrm{ml} / 1$ than untreated plants. Increasing the concentration of the protein hydrolysate from 0 to $10 \mathrm{ml} / 1$ increased the total dry biomass, SPAD index, and leaf nitrogen content by $20.5,15$, and $21.5 \%$, respectively. So, the use of plant-derived protein hydrolysate contain amino acids and short peptides excited a plant hormone-like activity, augmented nitrogen uptake and consequently crop performances (Colla et al., 2014). Amino acids for the production of bio-stimulants are obtained by chemical synthesis, from plant protein as well as from nimal proteins by chemical or enzymatic hydrolysis (Popko et al., 2014). Amino acids are considered as precursors and constituents of proteins (Rai, 2002), which are important for stimulation of cell growth. They contain both acidic and basic groups and work as buffers, which assist to protect appropriate $\mathrm{pH}$ value within the plant cell (Khan et al., 2012). Also, amino acids is a well-known bio-stimulants which has favorable effects on plant yield, growth and significantly moderates the damage raised by abiotic stresses (Kowalczyk et al., 2008). El-Zohiri and Asfour (2009) on potato recordrd that spraying of amino acids $(0.25 \mathrm{ml} / 1)$ increased vegetative growth expressed as plant height and plant dry weight. Therefore, the objective of the current research was to study the effect of foliar spray with bio-stimulants activity of native and modified (chemically and enzymatically) egg albumin on growth and output of pea plants under sandy soil conditions.

\section{MATERIALS AND METHODS}

\section{Experimental Design}

This research was carried out during the two successive winter seasons of 2015/ 2016 and 2016/2017 under sandy soil conditions using drip irrigation system at El-Khattara Experimental Farm, Faculty of Agriculture, Zagazig University, Sharkia Governorate, Egypt, to study the effect of native and modified egg white protein and total amino acids at different concentrations as foliar applications on growth, antioxidants, yield and seeds quality of pea cv. Master B. The physical and chemical properties of experimental soil in the two seasons showed that it was sandy in texture and had 0.07 and $0.08 \%$ organic matter, 8.03 and $8.08 \mathrm{pH}, 1.74$ and $1.76 \mathrm{mmhos} / \mathrm{cm} \mathrm{EC}, 5.35$ and $5.58 \mathrm{ppm}$ available N, 3.54 and 3.48 ppm available $\mathrm{P}$ and 9.879 .83 ppm available $\mathrm{K}$ in the $1^{\text {st }}$ and $2^{\text {nd }}$ season, respectively. 
This experiment included 16 treatments, which were the different concentrations (1.0, 1.5 and $2.0 \mathrm{ml} / 1$.) of native egg albumin (NEA), hydrolyzed native egg albumin (HNEA), methylated egg albumin (MEA), hydrolyzed methylated egg albumin (HMEA) and total amino acids (24\%) as foliar applications as well as control (water). These treatments were arranged in a randomized complete block design with three replications. Seeds were inoculated with Rhizobactrin at $400 \mathrm{~g} /$ fad., before sowing. Seeds sown at $5 \mathrm{~cm}$ apart, on $5^{\text {th }}$ October in both seasons and Master B cultivar was used in this experiment. The plot area was $12.6 \mathrm{~m}^{2}$. It contains three dripper lines with $6 \mathrm{~m}$ length each and 70 $\mathrm{cm}$ distance between each two dripper lines. One line was used to measure the morphological and physiological traits and other two lines were used for yield determinations. Plants were sprayed with the different concentrations of native and modified egg white protein and multi amino acids or tap water three time at 10 days intervals beginning 25 days after sowing in both seasons 25, 35 and 45 days after sowing. Each experimental plot received two liter solutions of each concentrations using spreading agent in all treatments to improve adherence of the spray to the plant foliage for increasing egg white protein absorption by the plants. The untreated plants (check) were sprayed with tap water and spreading agent. One dripper line was left between each two experimental plots without spraying as a gourd row to avoid the overlapping of spraying solution.

\section{Substances Preparation}

\section{Sample preparation}

Fresh hen eggs were collected from the Farm of Poultry Department, Faculty of Agriculture, Zagazig University. Protein extraction was performed basically according to the method reported by (Omana et al., 2011), with some modification. Egg white was accurately separated from yolk and softly homogenized with a magnetic stirrer for $30 \mathrm{~min}$ to decrease the viscosity. The white homogenate was lyophilized and stored at $-20^{\circ} \mathrm{C}$ until used. SDS-PAGE of protein was performed on a discontinuous buffered system according to (Laemmli, 1970). Total amino acids composition of egg white protein was determined by amino acid analyzer apparatus model "Eppendorf LC3000" (Simpson et al., 1976).

\section{Protein modification}

Lyophilized NEA was subjected to enzymatic modification by papain from $C$. papaya L (Sigma-St. Louis, MO, USA) and chemical modification (esterification reaction with methanol in the presence of $\mathrm{HCl}$ ). Also, MEA was subjected to chemical modification with methanol.

\section{Chemical modification}

The procedure of (Sitohy et al., 2000) was utilized for esterifying protein by dispersing a appropriate amount $(5 \% \mathrm{~W} / \mathrm{V})$ in concentrated methanol $(>99.5 \%)$. The reaction mix was preserved at $4{ }^{\circ} \mathrm{C}$ under continued stirring for $10 \mathrm{hr}$., while adding an amount of $\mathrm{HCl}$ equivalent to 50 molar ratio (mole acid $/ \mathrm{mole}-\mathrm{COOH})$ at the reaction start. The esterification extent of proteins was quantified according to (BertrandHarb et al., 1991).

\section{Enzymatic modification}

Lyophilized protein (NEA and MEA) was dissolved in $0.1 \mathrm{M}$ phosphate buffer $\mathrm{pH}$ $6.0(5 \%)$ and hydrolysed by treating with papain $(\mathrm{E} / \mathrm{S}$ ratio of $1: 200(\mathrm{~W} / \mathrm{W}))$ at $37^{\circ} \mathrm{C}$ for $4 \mathrm{hr}$., and $\mathrm{pH} 6.0$ as described by (Abdel-Hamid et al., 2016). The degree of hydrolysis was assayed according to the method described by Hoyle and Merrltt (1994).

\section{Data Recorded}

\section{Plant growth characters}

Five plants from each plot were randomly taken at 55 days after sowing and the following data were recorded: Plant growth: Plant height, branch number/plant, number of leaves/plant and total fresh weight/plant. 
Leaf pigments: disks sample from the fourth upper leaf was obtained from every experimental unit at 55 days after swing to determine chlorophyll $\mathrm{a}, \mathrm{b}$ and carotenoids according to the method described by Orsenigo and Marziani (1971).

Yield and its components: green pods were harvested at proper maturity counted and weighted in each harvest, and the following data were obtained: average number of green pods/plant, average green pod weight, number of seeds/plant, yield of green pods/plant and total yield of green pods/faddan.

Seed chemical constituent: A random sample of the dried green seeds was finely ground and wet digested and total nitrogen was determined according to Yimer et al., (2007). Phosphorus was estimated colorimetrically according to Olsen and Sommers (1982). Potassium was also determined flame photometrically due to the method described by Jackson (1970).

\section{RESULTS AND DISCUSSION}

\section{Protein Characterization}

When hen egg white albumin analyzed by SDS-PAGE (Fig. 1), egg white proteins presented a wide range of relative molecular masses, present in very different concentrations. The main egg white proteins, ovalbumin (54\%), ovotransferrin $(12 \%)$, ovomucoid $(11 \%)$ and lysozyme $3.4 \%$ ) these results are in harmony with (Mine, 1995).

The amino acids composition of hen egg white were listed in Table 1. The contents of the hydrophobic amino acids residues (Pro, Gly, Ala, Val, Ile, Leu, Phe) are $34.54 \%$ of the total amino acids. The contents of the acidic amino acid residues (asp + glu, are 22.25 and the case lower than that of the basic amino acids (arg + lys + his; $25.82 \%$.

Hen egg white protein was esterified with methanol in the presence of hydrochloric acid at $50 \mathrm{~mol}$ acid/ mole carboxylic groups, for $10 \mathrm{hr}$., at $4^{\circ} \mathrm{C}$ to attain esterification extents of $93 \%$. The extent of protein degradation (native and methylated) by papain was estimated by assessing the degree of hydrolysis (DH). The hydrolysate obtained after $4 \mathrm{hr}$., degradation had the highest $\mathrm{DH}$ (33\% and $30 \%$ ) for NEA and MEA, respectively).

\section{Vegetative Growth}

As for stem length, results in Table 2 show that spraying pea plants with NEA or with MEG at $1,1.5$ and $2 \mathrm{ml} / 1$ or with HNEA at $1,1.5$ and $2 \mathrm{ml} / 1$ and with HMEA at $1 \mathrm{ml} / \mathrm{l}$ gave the highest stem length compared to the other treatments. Concerning number of branches/plant and total fresh weight/plant, spraying with NEA at $2 \mathrm{ml} / 1$ increased number of branches/plant and total fresh weight in both seasons with no significant differences with MEA at $2 \mathrm{ml} / 1$ with respect to number of branches/plant. As for number of leaves/plant, spraying pea plants with HNEA at 1.5 or $2 \mathrm{ml} / 1$ increased number of leaves/plant in both seasons. From the foregoing results it could be concluded that, NEA at $2 \mathrm{ml} / 1$ increased stem length, number of branches/plant and total fresh weight /plant, whereas HNEA at 1.5 or $2 \mathrm{ml} / 1$ increased number of leaves/plant. Also, spraying pea plants with modified egg white protein at afferent concentration increased vegetative growth compared to control (spraying with tap water). Two PHFs increased root and leaf growth and induced morphological changes in root architecture. Besides, the treatments increased NR and GS activities, indicating a positive function of the two hydrolyzates in the inducement of nitrate conversion into organic nitrogen (Ertani et al., 2009). The shoot, root dry weight, root area, and root length were significantly higher by 21,35 , 26 , and $24 \%$, respectively, in tomato processed plants with the protein hydrolysate at $6 \mathrm{~m} \mathrm{1/1}$ than unprocessed plants. Rising the concentration of the protein hydrolysate from 0 to $10 \mathrm{~m} 1 / 1$ 


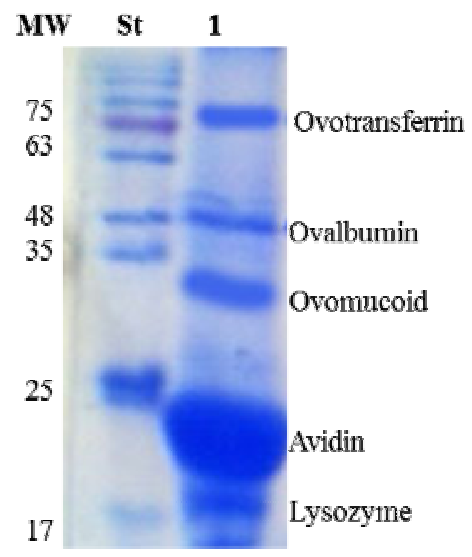

Fig. 1. SDS-PAGE of hen egg white protein (Lane 1) compared to standard molecular weight (St)

Table 1. Amino acid composition of hen egg white.

\begin{tabular}{lc}
\hline Amino acid & Concentration (\%) \\
\hline Aspartic & 9.39 \\
Threonine & 2.97 \\
Serine & 5.44 \\
Glutamic & 12.86 \\
Proline & 0.11 \\
Glycine & 7.56 \\
Alanine & 7.30 \\
Valine & 3.58 \\
Methionine & 4.14 \\
Isoleucine & 1.64 \\
Leucine & 6.10 \\
Tyrosine & 4.87 \\
Phenylalanine & 8.25 \\
Histidine & 4.12 \\
Lysine & 21.58 \\
Arginine & 0.12 \\
\hline
\end{tabular}


Table 2. Effect of foliar spray of pea plants with modified egg white proteins on vegetative growth of pea during $2015 / 2016$ and $2016 / 2017$ seasons

\begin{tabular}{|c|c|c|c|c|c|c|c|c|c|}
\hline \multirow{2}{*}{\multicolumn{2}{|c|}{ Treatment }} & \multirow{2}{*}{\multicolumn{2}{|c|}{$\begin{array}{l}\text { Stem length } \\
\text { (cm) }\end{array}$}} & \multirow{2}{*}{\multicolumn{2}{|c|}{$\begin{array}{c}\text { Number of } \\
\text { branches/plant }\end{array}$}} & \multirow{2}{*}{\multicolumn{2}{|c|}{$\begin{array}{c}\text { Number of } \\
\text { leaves/plant }\end{array}$}} & \multirow{2}{*}{\multicolumn{2}{|c|}{$\begin{array}{c}\text { Total fresh } \\
\text { weight/plant (g) }\end{array}$}} \\
\hline & & & & & & & & & \\
\hline & & \multirow{2}{*}{$\begin{array}{c}\mathbf{1}^{\text {st }} \\
\text { season } \\
54.33 \mathrm{de}\end{array}$} & $\begin{array}{c}2^{\text {nd }} \\
\text { season }\end{array}$ & $\begin{array}{c}1^{\text {st }} \\
\text { season }\end{array}$ & $\begin{array}{c}2^{\text {nd }} \\
\text { season }\end{array}$ & $\begin{array}{c}1^{\text {st }} \\
\text { season }\end{array}$ & $\begin{array}{c}2^{\text {nd }} \\
\text { season }\end{array}$ & $\begin{array}{c}1^{\text {st }} \\
\text { season }\end{array}$ & $\begin{array}{c}2^{\text {nd }} \\
\text { season }\end{array}$ \\
\hline \multicolumn{2}{|c|}{$\overline{\text { Control }}$} & & $55.33 \mathrm{e}$ & $1.33 \mathrm{fg}$ & $1.08 \mathrm{~d}$ & $13.00 \mathrm{e}$ & $13.00 \mathrm{j}$ & $31.16 \mathrm{f}$ & $28.33 \mathrm{~g}$ \\
\hline \multirow[t]{3}{*}{ NEA } & $1.0 \mathrm{ml} / \mathrm{l}$ & $56.00 \mathrm{c}-\mathrm{e}$ & $56.66 \mathrm{de}$ & $2.00 \mathrm{c}-\mathrm{e}$ & $1.83 b c$ & $18.00 \mathrm{bc}$ & $20.33 \mathrm{a}-\mathrm{c}$ & $72.66 \mathrm{bc}$ & $72.66 \mathrm{~b}$ \\
\hline & $1.5 \mathrm{ml} / \mathrm{l}$ & $60.33 \mathrm{a}-\mathrm{c}$ & $62.33 \mathrm{a}-\mathrm{c}$ & $1.75 \mathrm{ef}$ & $1.83 \mathrm{bc}$ & $17.66 \mathrm{~b}-\mathrm{d}$ & $18.00 \mathrm{c}-\mathrm{f}$ & $36.33 \mathrm{ef}$ & $38.16 \mathrm{e}$ \\
\hline & $2.0 \mathrm{ml} / \mathrm{l}$ & $62.00 \mathrm{ab}$ & $63.66 \mathrm{ab}$ & $3.08 \mathrm{a}$ & $2.58 \mathrm{a}$ & $14.33 \mathrm{e}$ & $14.00 \mathrm{ij}$ & $80.00 \mathrm{a}$ & $85.66 \mathrm{a}$ \\
\hline \multirow[t]{3}{*}{ HNEA } & $1.0 \mathrm{ml} / \mathrm{l}$ & $65.33 \mathrm{a}$ & $63.33 \mathrm{ab}$ & $2.50 \mathrm{bc}$ & $2.00 \mathrm{a}-\mathrm{c}$ & $23.00 \mathrm{a}$ & $22.66 \mathrm{a}$ & $73.33 \mathrm{~b}$ & $73.66 \mathrm{~b}$ \\
\hline & $1.5 \mathrm{ml} / \mathrm{l}$ & $61.00 \mathrm{a}-\mathrm{c}$ & $61.66 \mathrm{a}-\mathrm{d}$ & $1.25 \mathrm{fg}$ & $1.16 \mathrm{~d}$ & $22.33 \mathrm{a}$ & $21.66 \mathrm{ab}$ & $31.33 \mathrm{f}$ & $29.33 \mathrm{~g}$ \\
\hline & $2.0 \mathrm{ml} / \mathrm{l}$ & $a b$ & $64.66 \mathrm{a}$ & $1.91 \mathrm{de}$ & $1.83 \mathrm{bc}$ & $14.33 \mathrm{e}$ & $15.33 \mathrm{~g}-\mathrm{j}$ & $71.33 \mathrm{bc}$ & $73.00 \mathrm{~b}$ \\
\hline \multirow[t]{3}{*}{ MEA } & $1.0 \mathrm{ml} / \mathrm{l}$ & 57.0 & $57.33 \mathrm{c}-\mathrm{e}$ & $2.00 \mathrm{c}-\mathrm{e}$ & $1.91 \mathrm{bc}$ & $18.00 \mathrm{bc}$ & $17.00 \mathrm{e}-\mathrm{h}$ & $58.50 \mathrm{~d}$ & $57.33 \mathrm{~d}$ \\
\hline & 1.5 & 60 & e & 2. & $d$ & $b$ & $18.66 \mathrm{c}-\mathrm{e}$ & $38.00 \mathrm{e}$ & 35.6 \\
\hline & 2. & 6 & $63.00 \mathrm{ab}$ & 3. & $\mathrm{~b}$ & $\mathrm{~b}$ & 17.3 & $\mathrm{c}$ & $65.66 \mathrm{c}$ \\
\hline \multirow[t]{3}{*}{ HMEA } & $1.0 \mathrm{ml} / \mathrm{l}$ & $a-c$ & 62.6 & 1.7 & 1.9 & 18. & 19.6 & $57.66 \mathrm{~d}$ & $62.33 \mathrm{~cd}$ \\
\hline & $1.5 \mathrm{ml} / \mathrm{l}$ & $57.33 \mathrm{~b}-\mathrm{d}$ & $57.33 \mathrm{c}-\mathrm{e}$ & $1.91 \mathrm{de}$ & $1.91 \mathrm{bc}$ & $19.00 \mathrm{~b}$ & $19.66 \mathrm{~b}-\mathrm{d}$ & $31.16 \mathrm{f}$ & $31.33 \mathrm{fg}$ \\
\hline & $2.0 \mathrm{ml} / \mathrm{l}$ & $58.00 \mathrm{~b}-\mathrm{d}$ & $58.66 \mathrm{~b}-\mathrm{e}$ & $2.33 \mathrm{~cd}$ & $2.41 \mathrm{ab}$ & $15.33 \mathrm{c}-\mathrm{e}$ & $15.66 \mathrm{f}-\mathrm{i}$ & $74.00 \mathrm{~b}$ & $74.66 \mathrm{~b}$ \\
\hline \multirow[t]{3}{*}{ MAA } & $1.0 \mathrm{ml} / \mathrm{l}$ & $50.66 \mathrm{e}$ & $49.66 \mathrm{f}$ & $1.16 \mathrm{~g}$ & $1.91 \mathrm{bc}$ & $17.66 \mathrm{~b}-\mathrm{d}$ & $17.00 \mathrm{e}-\mathrm{h}$ & $40.33 \mathrm{e}$ & $38.33 \mathrm{e}$ \\
\hline & $1.5 \mathrm{ml} / \mathrm{l}$ & $58.00 \mathrm{~b}-\mathrm{d}$ & $57.33 \mathrm{c}-\mathrm{e}$ & $2.08 \mathrm{c}-\mathrm{e}$ & $1.91 \mathrm{bc}$ & $15.00 \mathrm{de}$ & $14.66 \mathrm{~h}-\mathrm{j}$ & $31.66 \mathrm{f}$ & $31.66 \mathrm{fg}$ \\
\hline & $2.0 \mathrm{ml} / \mathrm{l}$ & $58.00 \mathrm{~b}-\mathrm{d}$ & $57.33 \mathrm{c}-\mathrm{e}$ & $1.33 \mathrm{fg}$ & $1.41 \mathrm{~cd}$ & $9.00 \mathrm{f}$ & $8.00 \mathrm{k}$ & $38.66 \mathrm{e}$ & $37.33 \mathrm{e}$ \\
\hline \multicolumn{2}{|c|}{ LSD at 0.05 level } & 5.82 & 5.45 & 0.54 & 0.64 & 2.68 & 2.38 & 5.55 & 5.14 \\
\hline
\end{tabular}

Control = tap water (NEAative control), NEA= Native egg Albumin, HNEA= Hydrolyzed Native egg Albumin MEA=Methylated egg Albumin, HMEA= Hydrolyzed methylated egg Albumin, MAA= Multi Amino acids 24\% (positive control)

increased value the total dry biomass, SPAD index, and leaf nitrogen content by $20.5,15$, and $21.5 \%$, respectively. So, the use of plant-derived protein hydrolysate contain amino acids and short peptides excited a plant hormone-like activity, augmented nitrogen uptake and consequently crop performances (Colla et al., 2014).

\section{Leaf Pigments}

Results in Table 3 show that all treatments increased the concentration of chlorophyll $\mathrm{a} . \mathrm{b}$ and $(\mathrm{a}+\mathrm{b})$ and carotenoids in leaf tissues compared to control (tap water). Foliar spray with HMEA at $2 \mathrm{ml} / \mathrm{l}$ increased the concentration of chlorophyll $\mathrm{a}, \mathrm{b}$, and $(\mathrm{a}+\mathrm{b})$ and carotenoids compared to other treatments with no significant different with NEA, HNEA and MAA at 2 $\mathrm{ml} / \mathrm{l}$ of each with respect to chlorophyll $b$ in both seasons. Increasing the concentration of the protein hydrolysate from 0 to $10 \mathrm{ml} / 1$ increased the total dry biomass, SPAD index, and leaf nitrogen content by 20.5, 15, and $21.5 \%$, respectively. So, the use of plant-derived protein hydrolysate contain amino acids and short peptides excited a plant hormone-like activity, augmented nitrogen uptake and consequently crop performances (Colla et al., 2014). 
SINAI Journal of Applied Sciences (ISSN: 2314-6079), Vol. (6), Is. (1), Apr. 2017

Table 3. Effect of foliar spray of pea plants with modified egg white proteins on chlorophyll a,b and total chlorophyll $(a+b)$ and carotenoids during 2015/2016 and 2016/2017 seasons

\begin{tabular}{|c|c|c|c|c|c|c|c|c|c|}
\hline & & \multicolumn{2}{|c|}{ Chlorophyll a } & \multicolumn{2}{|c|}{ Chlorophyll b } & \multicolumn{2}{|c|}{$\begin{array}{c}\begin{array}{c}\text { Total Chlorophyll } \\
(\mathrm{a}+\mathrm{b})\end{array} \\
\end{array}$} & \multicolumn{2}{|c|}{ Carotenoids } \\
\hline & & S1 & S2 & S1 & S2 & S1 & S2 & S1 & S2 \\
\hline \multicolumn{2}{|l|}{$\overline{\text { Control }}$} & $1.254 \mathrm{fg}$ & g $1.276 \mathrm{gh}$ & $0.455 \mathrm{~g}$ & $0.480 \mathrm{i}$ & $1.709 \mathrm{~h}$ & $1.756 \mathrm{i}$ & $0.318 \mathrm{j}$ & $0.336 \mathrm{~g}$ \\
\hline \multirow[t]{3}{*}{ NEA } & $\mathrm{ml} / \mathrm{l}$ & $1.282 \mathrm{fg}$ & $\mathrm{g} 1.280 \mathrm{gh}$ & 0.520 ef & $0.507 \mathrm{hi}$ & $1.802 \mathrm{fg}$ & $1.788 \mathrm{hi}$ & $0.369 \mathrm{~g}$ & $0.337 \mathrm{~g}$ \\
\hline & $1.5 \mathrm{ml} / \mathrm{l}$ & $1.375 \mathrm{~d}$ & $1.334 \mathrm{~d}-\mathrm{f}$ & $0.636 \mathrm{~cd}$ & $0.609 \mathrm{de}$ & $2.011 \mathrm{~d}$ & $1.943 \mathrm{ef}$ & $0.381 \mathrm{fg}$ & $0.369 \mathrm{f}$ \\
\hline & $2.0 \mathrm{ml} / \mathrm{l}$ & $1.577 \mathrm{bc}$ & $1.614 \mathrm{~b}$ & $0.752 \mathrm{a}$ & $0.745 \mathrm{a}$ & $2.330 \mathrm{bc}$ & $2.359 \mathrm{bc}$ & $0.529 \mathrm{c}$ & $0.526 \mathrm{c}$ \\
\hline \multirow[t]{3}{*}{ HNEA } & $1.0 \mathrm{ml} / \mathrm{l}$ & $1.260 \mathrm{fg}$ & $1.260 \mathrm{~h}$ & 0.541 ef & $0.520 \mathrm{gh}$ & $1.802 \mathrm{fg}$ & $1.781 \mathrm{hi}$ & $0.343 \mathrm{hi}$ & $0.339 \mathrm{~g}$ \\
\hline & $1.5 \mathrm{ml} / \mathrm{l}$ & $1.356 \mathrm{~d}$ & $1.371 \mathrm{~d}$ & $0.640 \mathrm{~b}-\mathrm{d}$ & $0.657 \mathrm{bc}$ & $1.997 \mathrm{~d}$ & $2.029 \mathrm{~d}$ & $0.393 \mathrm{ef}$ & $0.391 \mathrm{de}$ \\
\hline & $2.0 \mathrm{ml} / \mathrm{l}$ & $1.581 \mathrm{bc}$ & $1.617 \mathrm{~b}$ & $0.756 \mathrm{a}$ & $0.760 \mathrm{a}$ & $2.337 \mathrm{bc}$ & $2.377 \mathrm{~b}$ & $0.556 \mathrm{~b}$ & $0.541 b c$ \\
\hline \multirow[t]{3}{*}{ MEA } & $1.0 \mathrm{ml} / \mathrm{l}$ & $1.289 \mathrm{e}-\mathrm{g}$ & g $1.293 \mathrm{f}-\mathrm{h}$ & $0.546 \mathrm{e}$ & $0.550 \mathrm{fg}$ & $1.836 \mathrm{f}$ & $1.843 \mathrm{gh}$ & $0.364 \mathrm{gh}$ & $0.345 \mathrm{~g}$ \\
\hline & $1.5 \mathrm{ml} / \mathrm{l}$ & $1.351 \mathrm{~d}$ & $1.377 \mathrm{~d}$ & $0.654 \mathrm{bc}$ & $0.662 \mathrm{bc}$ & $2.005 \mathrm{~d}$ & $2.039 \mathrm{~d}$ & $0.413 \mathrm{de}$ & $0.398 \mathrm{~d}$ \\
\hline & $2.0 \mathrm{ml} / \mathrm{l}$ & $1.616 \mathrm{~b}$ & $1.655 \mathrm{~b}$ & $0.760 \mathrm{a}$ & $0.775 \mathrm{a}$ & $2.377 \quad b$ & $2.430 \mathrm{~b}$ & $0.571 \mathrm{~b}$ & $0.553 \mathrm{~b}$ \\
\hline \multirow[t]{3}{*}{ HMEA } & $1.0 \mathrm{ml} / \mathrm{l}$ & 1.298 ef & $1.320 \mathrm{e}-\mathrm{g}$ & $0.610 \mathrm{~d}$ & $0.573 \mathrm{ef}$ & $1.908 \mathrm{e}$ & $1.894 \mathrm{fg}$ & $0.373 \mathrm{fg}$ & 0.378 ef \\
\hline & $1.5 \mathrm{ml} / \mathrm{l}$ & $1.333 \mathrm{de}$ & $1.330 \mathrm{~d}-\mathrm{f}$ & $0.671 \mathrm{~b}$ & $0.676 \mathrm{~b}$ & $2.005 \mathrm{~d}$ & $2.006 \mathrm{de}$ & $0.422 \mathrm{~d}$ & $0.391 \mathrm{de}$ \\
\hline & $2.0 \mathrm{ml} / \mathrm{l}$ & $1.735 \mathrm{a}$ & $1.759 \mathrm{a}$ & $0.757 \mathrm{a}$ & $0.765 \mathrm{a}$ & $2.493 \mathrm{a}$ & $2.524 \mathrm{a}$ & $0.601 \mathrm{a}$ & $0.637 \mathrm{a}$ \\
\hline \multirow[t]{3}{*}{ MAA } & $1.0 \mathrm{ml} / \mathrm{l}$ & $1.252 \mathrm{~g}$ & $1.264 \mathrm{~h}$ & $0.511 \mathrm{f}$ & $0.530 \mathrm{gh}$ & $1.763 \mathrm{gh}$ & $1.795 \mathrm{hi}$ & $0.335 \mathrm{ij}$ & $0.334 \mathrm{~g}$ \\
\hline & $1.5 \mathrm{n}$ & 1.35 & $1.350 \mathrm{de}$ & $0.634 \mathrm{~cd}$ & $0.625 \mathrm{~cd}$ & $1.989 \mathrm{~d}$ & $1.975 \mathrm{de}$ & $0.365 \mathrm{~g}$ & $0.381 \mathrm{~d}-\mathrm{f}$ \\
\hline & $2.0 \mathrm{ml} / \mathrm{l}$ & $1.560 \mathrm{c}$ & $1.542 \mathrm{c}$ & $0.753 \mathrm{a}$ & $0.748 \mathrm{a}$ & $2.313 \mathrm{c}$ & $2.290 \mathrm{c}$ & $0.571 \mathrm{~b}$ & $0.537 \mathrm{bc}$ \\
\hline \multicolumn{2}{|c|}{ LSD at 0.05 level } & 0.044 & 0.047 & 0.033 & 0.037 & 0.054 & 0.071 & 0.021 & 0.020 \\
\hline
\end{tabular}

Control = tap water (NEAative control), NEA= Native egg Albumin, HNEA= Hydrolyzed Native egg Albumin $\mathrm{MEA}=$ Methylated egg Albumin, HMEA= Hydrolyzed methylated egg Albumin, MAA= Multi Amino acids $24 \%$ (positive control)

\section{Yield and its Components}

The obtained results in Table 4 show that spraying pea plants with modified egg weight protein at different concentrations increased yield and its components compared to control (spraying with tap water) Spraying with NEA at $1.5 \mathrm{ml} / 1$ or with HNEA at $1 \mathrm{ml} / 1$ significantly increased number of green pods/plant, average green pod, yield of green pods/plant and total yield of green pods/fad. in both seasons with no significant differences with NEA at $1 \mathrm{ml} / 1$ and MEA at $2 \mathrm{ml} / 1$ with respect to number of green pods/plant, MAA at 1.5 $\mathrm{ml} / \mathrm{l}$ and HNEA at $2 \mathrm{ml} / \mathrm{l}$ with respect to average green pod weight, and with NEA at $1 \mathrm{ml} / \mathrm{l}, \mathrm{HNEA}$ at $2 \mathrm{ml} / \mathrm{l}$ and MEA at $2 \mathrm{ml} / 1$ with respect to yield of green pods/plant. As for number of seeds/pod, NEA at 1.5 $\mathrm{ml} / 1$ and HMEA at 1.5 and $2 \mathrm{ml} / 1$ and MAA at $1.5 \mathrm{ml} / 1$ increased number of seeds/pod in both seasons. The simulative effect of NEA at $1.5 \mathrm{ml} / 1$ and HNEA at $1 \mathrm{ml} / 1$ on total yield of green pods/fad. may be due to that NEA and HNEA at $1.5 \mathrm{ml} / 1$ of each increased number of green pods/plant, average green pod weight and yield of green pods/plant. Similarly, Calvo et al. (2014) proposed that the using (through foliage or roots) of a $\mathrm{pH}$ trade product (Amino 16) could increase greenhouse tomato yield components under varying fertilization dosages. It is rather evident that the using of amino acids. 
Table 4. Effect of foliar spray of pea plants with modified egg white proteins on peas yield and its components during 2015/2016 and 2016/2017 seasons

\begin{tabular}{|c|c|c|c|c|c|c|c|c|c|c|c|}
\hline \multicolumn{2}{|c|}{ Treatment } & \multicolumn{2}{|c|}{$\begin{array}{l}\text { Number of green } \\
\text { pods/ plant }\end{array}$} & \multicolumn{2}{|c|}{$\begin{array}{l}\text { Average green pod } \\
\text { weight (g) }\end{array}$} & \multicolumn{2}{|c|}{$\begin{array}{l}\text { Number of seeds/ } \\
\text { pod }\end{array}$} & \multicolumn{2}{|c|}{$\begin{array}{l}\text { Yield of green pods / } \\
\text { plant (g) }\end{array}$} & \multicolumn{2}{|c|}{$\begin{array}{l}\text { Total yield of green } \\
\text { pods/fad. (ton) }\end{array}$} \\
\hline & & \multirow{2}{*}{$\frac{\mathbf{1}^{\text {st }} \text { season }}{8.33 \mathrm{~h}}$} & \multirow{2}{*}{$\frac{\mathbf{2}^{\text {nd }} \text { season }}{8.66 \mathrm{~g}}$} & \multicolumn{2}{|c|}{$1^{\text {st }} \operatorname{season} 2^{\text {nd }}$ season } & \multicolumn{2}{|c|}{$1^{\text {st }}$ season $2^{\text {nd }}$ season } & \multirow{2}{*}{$\begin{array}{c}\mathbf{1}^{\text {st }} \text { season } \\
51.10 \mathrm{~g}\end{array}$} & \multirow{2}{*}{$\begin{array}{c}\mathbf{2}^{\text {nd }} \text { season } \\
53.87 \mathrm{~g}\end{array}$} & \multicolumn{2}{|c|}{$1^{\text {st }}$ season $2^{\text {nd }}$ season } \\
\hline$\overline{\text { Control }}$ & & & & $6.13 \mathrm{a}-\mathrm{c}$ & 6.20 a-e & $7.53 b-f$ & 7.60 a-e & & & 1.5001 & $\overline{1.5}$ \\
\hline \multirow[t]{3}{*}{ NEA } & $1.0 \mathrm{ml} / \mathrm{l}$ & $19.66 \mathrm{ab}$ & $22.33 \mathrm{a}$ & $5.80 \mathrm{~b}-\mathrm{d}$ & $5.83 \mathrm{c}-\mathrm{g}$ & 7.43 & 7.501 & 114.13a-c & $130.07 \mathrm{ab}$ & $3.327 \mathrm{de}$ & $3.469 \mathrm{~d}$ \\
\hline & 1.5 & $20.00 \mathrm{a}$ & 20.33 & $a$ & 6. & 7.96 & 8 & 13 & 13 & $3.841 \mathrm{a}$ & $15 \mathrm{ab}$ \\
\hline & $2.0 \mathrm{ml} / \mathrm{l}$ & $18.00 \mathrm{a}-\mathrm{d}$ & 20.33a-c & $6.00 \mathrm{a}-\mathrm{c}$ & $5.96 \mathrm{~b}-\mathrm{f}$ & $7.43 \mathrm{c}-\mathrm{f}$ & 7.30 c-e & $108.40 \mathrm{a}-\mathrm{c}$ & $121.63 \mathrm{a}-\mathrm{c}$ & 3.185 ef & $3.567 \mathrm{~cd}$ \\
\hline \multirow[t]{3}{*}{ HNEA } & $1.0 \mathrm{ml} / \mathrm{l}$ & $18.66 \mathrm{a}-\mathrm{c}$ & $19.00 \mathrm{a}-\mathrm{d}$ & $6.06 \mathrm{a}-\mathrm{c}$ & $6.60 \mathrm{ab}$ & 7.60 a-e & $7.53 \mathrm{~b}-\mathrm{e}$ & $113.33 a-c$ & 125.3 & $3.789 \mathrm{ab}$ & $4.096 \mathrm{a}$ \\
\hline & $1.5 \mathrm{ml} / \mathrm{l}$ & $16.66 \mathrm{c}-\mathrm{f}$ & $17.00 \mathrm{c}-\mathrm{e}$ & $6.16 \mathrm{a}-\mathrm{c}$ & $6.13 \mathrm{a}-\mathrm{f}$ & $7.36 \mathrm{~d}-\mathrm{f}$ & 7.40 c-e & $102.23 b-d$ & $105.23 \mathrm{~b}-\mathrm{c}$ & $3.008 \mathrm{fg}$ & $2.773 \mathrm{hi}$ \\
\hline & $2.0 \mathrm{ml} / \mathrm{l}$ & 18.66 a-c & $19.00 \mathrm{a}-\mathrm{d}$ & $6.46 \mathrm{ab}$ & $6.70 \mathrm{a}$ & $7.20 \mathrm{ef}$ & $7.16 \mathrm{~d}-\mathrm{f}$ & $121.20 \mathrm{ab}$ & $127.67 \mathrm{ab}$ & $3.586 \mathrm{bc}$ & $3.763 \mathrm{bc}$ \\
\hline \multirow[t]{3}{*}{ MEA } & $1.0 \mathrm{ml} / \mathrm{l}$ & $14.66 \mathrm{e}-\mathrm{g}$ & $16.00 \mathrm{~d}-\mathrm{f}$ & $5.16 \mathrm{~d}$ & $5.16 \mathrm{~g}$ & $7.03 \mathrm{fg}$ & 7.06 ef & 75.67 ef & $83.00 \mathrm{ef}$ & $2.254 \mathrm{j}$ & $2.435 \mathrm{jk}$ \\
\hline & $1.5 \mathrm{ml} / \mathrm{l}$ & $14.66 \mathrm{e}-\mathrm{g}$ & $15.66 \mathrm{~d}-\mathrm{f}$ & $5.66 \mathrm{~cd}$ & $5.76 \mathrm{~d}-\mathrm{g}$ & $7.80 \mathrm{a}-\mathrm{d}$ & 7.86 a-c & $83.10 \mathrm{~d}-\mathrm{f}$ & $91.17 \mathrm{~d}-\mathrm{f}$ & $2.454 \mathrm{ij}$ & $2.685 \mathrm{~h}-\mathrm{j}$ \\
\hline & $2.0 \mathrm{ml} / \mathrm{l}$ & $20.00 \mathrm{a}$ & $20.66 \mathrm{ab}$ & $5.86 \mathrm{a}-\mathrm{c}$ & $5.60 \mathrm{e}-\mathrm{g}$ & $7.56 \mathrm{~b}-\mathrm{f}$ & 7.66 a-e & $117.20 \mathrm{ab}$ & $116.00 \mathrm{a}-\mathrm{d}$ & $3.439 \mathrm{~cd}$ & $3.410 \mathrm{de}$ \\
\hline \multirow[t]{3}{*}{ HMEA } & $1.0 \mathrm{ml} / \mathrm{l}$ & $16.00 \mathrm{c}-\mathrm{f}$ & $16.33 \mathrm{~d}-\mathrm{f}$ & $6.10 \mathrm{a}-\mathrm{c}$ & $6.03 \mathrm{a}-\mathrm{f}$ & $7.80 \mathrm{a}-\mathrm{d}$ & $8.03 \mathrm{ab}$ & $97.60 \mathrm{~b}-\mathrm{e}$ & $98.73 \mathrm{c}-\mathrm{f}$ & $2.375 \mathrm{j}$ & $2.807 \mathrm{hi}$ \\
\hline & $1.5 \mathrm{ml} / \mathrm{l}$ & $17.00 \mathrm{~b}-\mathrm{e}$ & $16.66 \mathrm{~d}-\mathrm{f}$ & 5.7 & $c-f$ & 8.0 & 8.1 & 97 & 98. & gh & $2.9277 \mathrm{gh}$ \\
\hline & $2.0 \mathrm{ml} / \mathrm{l}$ & 20.33 a & 18.66b-e & 5.7 & 5.7 & 7.6 & $7.73 \mathrm{a}-\mathrm{d}$ & 117.17ab & 107.07 & $3.449 \mathrm{~cd}$ & $3.145 \mathrm{fg}$ \\
\hline \multirow[t]{3}{*}{ MAA } & $1.0 \mathrm{ml} / \mathrm{l}$ & $15.66 \mathrm{~d}-\mathrm{g}$ & $15.33 \mathrm{ef}$ & $5.83 \mathrm{a}-\mathrm{d}$ & $5.66 \mathrm{e}-\mathrm{g}$ & $7.46 \mathrm{~b}-\mathrm{f}$ & 7.30 c-e & $91.40 \mathrm{c}-\mathrm{f}$ & $86.60 \mathrm{ef}$ & $2.670 \mathrm{hi}$ & $2.587 \mathrm{ij}$ \\
\hline & $1.5 \mathrm{ml} / \mathrm{l}$ & $14.00 \mathrm{fg}$ & $17.00 \mathrm{c}-\mathrm{e}$ & $6.46 \mathrm{ab}$ & $6.36 \mathrm{a}-\mathrm{d}$ & $8.13 \mathrm{a}$ & $8.20 \mathrm{a}$ & $90.63 \mathrm{c}-\mathrm{f}$ & 108.07 a-e & $2.665 \mathrm{i}$ & 3.195 ef \\
\hline & $2.0 \mathrm{ml} / \mathrm{l}$ & $13.00 \mathrm{~g}$ & $13.33 \mathrm{f}$ & $5.56 \mathrm{~cd}$ & $5.50 \mathrm{fg}$ & $6.53 \mathrm{~g}$ & $6.56 \mathrm{f}$ & $72.53 \mathrm{fg}$ & $73.47 \mathrm{fg}$ & $1.842 \mathrm{k}$ & $2.185 \mathrm{k}$ \\
\hline \multicolumn{2}{|c|}{ LSD at 0.05 level } & 2.82 & 3.46 & 0.69 & 0.69 & 0.54 & 0.61 & 24.15 & 27.53 & 0.253 & 0.253 \\
\hline
\end{tabular}

Control = tap water (NEAative control), NEA= Native egg Albumin, HNEA= Hydrolyzed Native egg Albumin MEA=Methylated egg Albumin, HMEA= Hydrolyzed methylated egg Albumin, MAA= Multi Amino acids 24\% (positive control)

\section{Seed Chemical Constituents}

Results in Table 5 illustrate that spraying with modified egg white protein at different concentration increased $\mathrm{N}, \mathrm{P}, \mathrm{K}$ and total protein in seeds compared to control (spraying with tap water). Spraying with NEA at $1.5 \mathrm{ml} / \mathrm{l}$ increased $\mathrm{N}, \mathrm{P}, \mathrm{K}$ and total protein in seeds in the $1^{\text {st }}$ season, whereas spraying with MAA at $2 \mathrm{ml} / 1$ increased $\mathrm{N}$ and total protein in seeds, MAA at $1 \mathrm{ml} / 1$ increased $\mathrm{P}$ content and HMEA at $2 \mathrm{ml} / \mathrm{l}$ increased $\mathrm{K}$ content in both seasons.
Several studies indicated that soil microorganisms used $30-40 \%$ of amino acid-C for respiration and the remaining amino acid-Cfor cell biomass production and maintenance (McMahon, 2008). Moreover, $30-40 \%$ of the $\mathrm{N}$ linked with the respired amino acid-C was consistently excreted into the soil as ammonium-N, which can be taken up by plants and microbes or further oxidised to produce nitrate. 
SINAI Journal of Applied Sciences (ISSN: 2314-6079), Vol. (6), Is. (1), Apr. 2017

Table 5. Effect of foliar spray of pea plants with modified egg white proteins on seed chemical content during 2015/2016 and 2016/2017 seasons

\begin{tabular}{|c|c|c|c|c|c|c|c|c|c|}
\hline \multicolumn{2}{|c|}{ Treatment } & \multicolumn{2}{|c|}{$\mathbf{N}(\%)$} & \multicolumn{2}{|c|}{$P(\%)$} & \multicolumn{2}{|c|}{ K (\%) } & \multicolumn{2}{|c|}{ Total protein (\%) } \\
\hline & & $\begin{array}{c}1^{\text {st }} \\
\text { season }\end{array}$ & $\begin{array}{c}2^{\text {nd }} \\
\text { season }\end{array}$ & $\begin{array}{c}1^{\text {st }} \\
\text { season }\end{array}$ & $\begin{array}{c}2^{\text {nd }} \\
\text { season }\end{array}$ & $\begin{array}{c}1^{\text {st }} \\
\text { season }\end{array}$ & $\begin{array}{c}2^{\text {nd }} \\
\text { season }\end{array}$ & $\begin{array}{c}1^{\text {st }} \\
\text { season }\end{array}$ & $\begin{array}{c}2^{\text {nd }} \\
\text { season }\end{array}$ \\
\hline \multicolumn{2}{|l|}{$\overline{\text { Control }}$} & $2.48 \mathrm{~cd}$ & $2.35 \mathrm{~h}$ & $0.513 \mathrm{c}-\mathrm{e}$ & $0.543 \mathrm{ab}$ & $1.94 \mathrm{~g}$ & $1.71 \mathrm{i}$ & $15.50 \mathrm{~cd}$ & $14.71 \mathrm{~h}$ \\
\hline \multirow[t]{3}{*}{ NEA } & $1.0 \mathrm{ml} / \mathrm{l}$ & $2.96 \mathrm{~b}$ & $2.62 \mathrm{f}$ & $0.500 \mathrm{e}$ & $0.490 \mathrm{~cd}$ & $1.98 \mathrm{f}$ & $1.88 \mathrm{~h}$ & $18.52 \mathrm{~b}$ & $16.39 \mathrm{f}$ \\
\hline & $1.5 \mathrm{ml} / \mathrm{l}$ & $3.33 \mathrm{a}$ & $2.95 \mathrm{e}$ & $0.556 \mathrm{a}$ & $0.510 \mathrm{~b}-\mathrm{d}$ & $2.40 \mathrm{a}$ & $2.10 \mathrm{~cd}$ & $20.81 \mathrm{a}$ & $18.46 \mathrm{e}$ \\
\hline & $2.0 \mathrm{ml} / \mathrm{l}$ & $2.54 \mathrm{c}$ & $3.25 \mathrm{a}-\mathrm{c}$ & $0.436 \mathrm{f}$ & $0.503 \mathrm{~b}-\mathrm{d}$ & $1.84 \mathrm{i}$ & $2.44 \mathrm{a}$ & 15.87 & $20.31 \mathrm{a}-\mathrm{c}$ \\
\hline \multirow[t]{3}{*}{ HNEA } & $1.0 \mathrm{ml} / \mathrm{l}$ & $3.07 \mathrm{ab}$ & $2.94 \mathrm{e}$ & $0.546 \mathrm{ab}$ & $0.530 \mathrm{a}-\mathrm{c}$ & $2.30 \mathrm{~b}$ & $2.10 \mathrm{~d}$ & $19.19 \mathrm{ab}$ & $18.37 \mathrm{e}$ \\
\hline & $1.5 \mathrm{ml} / \mathrm{l}$ & $2.23 \mathrm{~d}$ & $3.42 \mathrm{a}$ & $0.556 \mathrm{a}$ & $0.506 \mathrm{~b}-\mathrm{d}$ & $1.92 \mathrm{gh}$ & $2.16 \mathrm{c}$ & $13.93 \mathrm{~d}$ & $21.39 \mathrm{a}$ \\
\hline & $2.0 \mathrm{ml} / \mathrm{l}$ & $2.53 \mathrm{c}$ & $3.25 \mathrm{a}-\mathrm{c}$ & $0.510 \mathrm{de}$ & $0.483 \mathrm{~d}$ & $1.75 \mathrm{j}$ & $1.83 \mathrm{~h}$ & $15.81 \mathrm{c}$ & $20.33 \mathrm{a}-\mathrm{c}$ \\
\hline \multirow[t]{3}{*}{ MEA } & $1.0 \mathrm{ml} / \mathrm{l}$ & $3.30 \mathrm{a}$ & $3.09 \mathrm{c}-\mathrm{e}$ & $0.513 c-e$ & $0.510 \mathrm{~b}-\mathrm{d}$ & $1.74 \mathrm{j}$ & $2.11 \mathrm{~cd}$ & $20.62 \mathrm{a}$ & $19.35 \mathrm{c}-\mathrm{e}$ \\
\hline & $1.5 \mathrm{ml} / \mathrm{l}$ & $2.45 \mathrm{~cd}$ & $2.39 \mathrm{gh}$ & $0.513 \mathrm{c}-\mathrm{e}$ & $0.530 \mathrm{a}-\mathrm{c}$ & $1.89 \mathrm{~h}$ & $1.84 \mathrm{~h}$ & $15.33 \mathrm{~cd}$ & $14.98 \mathrm{gh}$ \\
\hline & $2.0 \mathrm{ml} / \mathrm{l}$ & $2.54 \mathrm{c}$ & $2.99 \mathrm{e}$ & $0.496 \mathrm{e}$ & $0.536 \mathrm{ab}$ & $1.91 \mathrm{gh}$ & $2.09 \mathrm{de}$ & $15.91 \mathrm{c}$ & $18.69 \mathrm{e}$ \\
\hline \multirow[t]{3}{*}{ HMEA } & $1.0 \mathrm{ml} / \mathrm{l}$ & $2.65 \mathrm{c}$ & $2.54 \mathrm{fg}$ & $0.513 \mathrm{c}-\mathrm{e}$ & $0.503 \mathrm{~b}-\mathrm{d}$ & $1.99 \mathrm{f}$ & $1.84 \mathrm{~h}$ & $16.56 \mathrm{c}$ & $15.89 \mathrm{fg}$ \\
\hline & $1.5 \mathrm{ml} / \mathrm{l}$ & $2.96 \mathrm{~b}$ & $3.04 \mathrm{de}$ & $0.516 \mathrm{~b}-\mathrm{e}$ & $0.556 \mathrm{a}$ & $2.10 \mathrm{e}$ & $1.96 \mathrm{~g}$ & $18.52 \mathrm{~b}$ & $19.02 \mathrm{de}$ \\
\hline & $2.0 \mathrm{ml} / \mathrm{l}$ & $3.22 \mathrm{ab}$ & $3.21 \mathrm{~b}-\mathrm{d}$ & $0.536 \mathrm{a}-\mathrm{d}$ & $0.433 \mathrm{e}$ & $2.44 \mathrm{a}$ & $2.44 \mathrm{a}$ & $20.14 \mathrm{al}$ & 20.06 b-d \\
\hline \multirow[t]{3}{*}{ MAA } & $1.0 \mathrm{ml} / \mathrm{l}$ & $3.22 \mathrm{ab}$ & $2.56 \mathrm{fg}$ & $0.533 a-d$ & $0.553 \mathrm{a}$ & $2.17 \mathrm{~d}$ & 2.03 ef & $20.14 \mathrm{ab}$ & $16.04 \mathrm{fg}$ \\
\hline & $1.5 \mathrm{ml} / \mathrm{l}$ & $2.97 \mathrm{~b}$ & $3.29 \mathrm{ab}$ & $0.543 a-c$ & $0.560 \mathrm{a}$ & $2.26 \mathrm{c}$ & $1.96 \mathrm{fg}$ & $18.56 \mathrm{~b}$ & $20.60 \mathrm{ab}$ \\
\hline & $2.0 \mathrm{ml} / \mathrm{l}$ & $3.23 \mathrm{a}$ & $3.25 \mathrm{a}-\mathrm{c}$ & $0.550 \mathrm{a}$ & $0.506 \mathrm{~b}-\mathrm{d}$ & $1.84 \mathrm{i}$ & $2.29 \mathrm{~b}$ & $20.23 \mathrm{a}$ & $20.35 a-c$ \\
\hline \multicolumn{2}{|c|}{ LSD at 0.05 level } & 0.26 & 0.18 & 0.031 & 0.40 & 0.03 & 0.06 & 1.66 & 1.17 \\
\hline
\end{tabular}

Control = tap water (NEAative control), NEA= Native egg Albumin, HNEA= Hydrolyzed Native egg Albumin MEA=Methylated egg Albumin, HMEA= Hydrolyzed Methylated egg Albumin, MAA= Multi Amino acids $24 \%$ (positive control)

\section{REFERENCES}

Abdel-Hamid, M.; Goda, H.A.; De Gobba, C.; Jenssen, H. and Osman, A. (2016). Antibacterial activity of papain hydrolysed camel whey and its fractions. Int. Dairy J., 61: 91-98.

Bertrand-Harb, C.; Chobert, J.M.; Dufour, E. and Haertle, T. (1991). Esterification of food proteins: Characterization of the derivatives by a colorimetric method and by electrophoresis. Sci. des Aliments (France).

Biesaga-Koscielniak, J.; Dziurka, M.; Ostrowska, A.; Mirek, M.;
Koscielniak, J. and Janeczko, A. (2014). Brassinosteroid improves content of antioxidants in seeds of selected leguminous plants. Aust. J. Crop Sci., 8 (3): 378 .

Calvo, P.; Nelson, L. and Kloepper, J.W. (2014). Agricultural uses of plant biostimulants. Plant and Soil, 383 (1-2): 3-41.

Colla, G.; Rouphael, Y.; Canaguier, R.; Svecova, E. and Cardarelli, M. (2014). Biostimulant action of a plant-derived protein hydrolysate produced through enzymatic hydrolysis. Frontiers in Plant Sci., 5: 448. 
El-Zohiri, S.S.M. and Asfour, Y.M. (2009). Effect of some organic compounds on growth and productivity of some potato cultivars. Ann. Agric. Sci. Moshtohor, 47(3): 403-415.

Ertani, A.; Cavani, L.; Pizzeghello, D.; Brandellero, E.; Altissimo, A.; Ciavatta, C. and Nardi, S. (2009). Biostimulant activity of two protein hydrolyzates in the growth and nitrogen metabolism of maize seedlings. J. Plant Nutr. and Soil Sci., 172 (2): 237-244.

Gurav, R.G. and J.P. Jadhav (2013). A novel source of biofertilizer from feather biomass for banana cultivation. Environ. Sci. and Pollution Res., 20 (7): 45324539.

Hoyle, N.T. and Merrltt, J.H. (1994). Quality of fish protein hydrolysates from herring (Clupea harengus). J. Food Sci., 59 (1): 76-79.

Ito, Y.; Nakanomyo, I.; Motose, H.; Iwamoto, K.; Sawa, S.; Dohmae, N. and Fukuda, H. (2006). Dodeca-CLE peptides as suppressors of plant stem cell differentiation. Sci., 313 (5788): 842845.

Jackson, M.L. (1970). Soil Chemical Analysis Prentice Hall. Englewood Gliffs, N.J.

Kauffman, G.L.; Kneivel, D.P. and Watschke, T.L. (2007). Effects of a biostimulant on the heat tolerance associated with photosynthetic capacity, membrane thermostability, and polyphenol production of perennial ryegrass. Crop Sci., 47 (1): 261-267.

Khan, A.S.; Ahmad, B.; Jaskani, M.J.; Rashid, A. and Malik, A.U. (2012). Foliar application of mixture of amino acids and seaweed (Ascophylum nodosum) extract improve growth and physicochemical properties of grapes. Int. J. Agric. and Biol., 14 (3): 210-219.
Kondo, T.; Sawa, S.; Kinoshita, A.; Mizuno, S.; Kakimoto, T.; Fukuda, H. and Sakagami, Y. (2006). A plant peptide encoded by CLV3 identified by in situ MALDI-TOF MS analysis. Sci., 313 (5788): 845-848.

Kowalczyk, K.; Zielony, T. and Gajewski, M. (2008). Effect of Aminoplant and Asahi on yield and quality of lettuce grown on rockwool. Biostimulators in Modern Agric. Veg. Crops, 35-43.

Laemmli, U.K. (1970). Cleavage of structural proteins during the assembly of the head of bacteriophage T4. Nat., 227 (5259): 680.

McMahon, S.K. (2008). Winter microbial carbon metabolism and community composition in Alaskan tundra soil. California Univ., Santa Barbara.

Mine, Y. (1995). Recent advances in the understanding of egg white protein functionality. Trends in Food Sci. and Technol., 6: 225-232.

Olsen, S.R and Sommers, L.E. (1982). Phosphorus. In: Page, AL, RH Miller and D.R. Keeney (Eds). Methods of Soil Analysis. Part 2, Ame. Soc. Agro. Madison, W.I. USA, 403-430.

Omana, D.A.; Liang, Y.; Kav, N.N. and Wu, J. (2011). Proteomic analysis of egg white proteins during storage. Proteomics, 11 (1): 144-153.

Orsenigo, M. and Marziani, G. (1971). Chloroplast Structural Changes During Temperature-Induced Bleaching and ReGreening in a «Golden Leaf» Mutant of Maize. Caryologia, 24 (3): 347-364.

Osman, A.; Goda, H.A.; Abdel-Hamid, M.; Badran, S.M. and Otte, J. (2016). Antibacterial peptides generated by Alcalase hydrolysis of goat whey. LWTFood Sci. and Technol., 65: 480-486. 
Pihlanto, A. and Korhonen, H. (2003). Bioactive peptides and proteins. Adv. Food and Nutr. Res., 47(4): 175-276.

Popko, M.; Wilk, R. and Gorecki, H. (2014). New amino acid biostimulators based on protein hydrolysate of keratin. Przemysl Chem., 93 (6): 10121015.

Poullain, M.G.; Cezard, J.P.; Roger, L. and Mendy, F. (1989). Effect of whey proteins, their oligopeptide hydrolysates and free amino acid mixtures on growth and nitrogen retention in fed and starved rats. J. Parenteral and Enteral Nutr., 13 (4): 382-386.

Rai, V.K. (2002). Role of amino acids in plant responses to stresses. Biologia Plantarum, 45 (4): 481-487.
Simpson, R.J.; Neuberger, M.R. and Liu, T.Y. (1976). Complete amino acid analysis of proteins from a single hydrolysate. J. Biol. Chem., 251 (7): 1936-1940.

Sitohy, M.; Chobert, J.M. and Haertle, T. (2000). Study of factors influencing protein esterification using $\beta$-lactoglobulin as a model. J. Food Biochem., 24 (5): 381-398.

Yimer, F.; Ledin, S. and Abdelkadir, A. (2007). Changes in soil organic carbon and total nitrogen contents in three adjacent land use types in the Bale Mountains, south-eastern highlands of Ethiopia. Forest Ecol. and Manag., 242 (2-3): 337-342. 


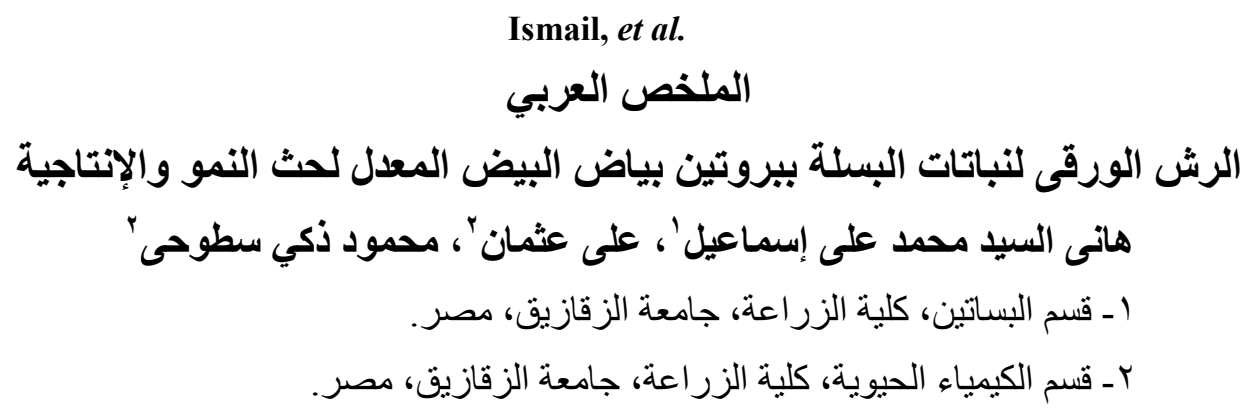

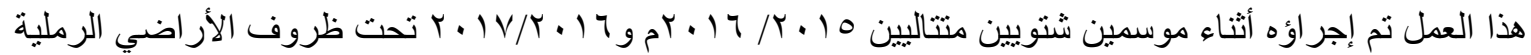

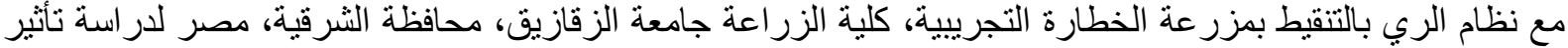

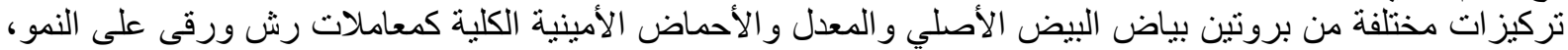

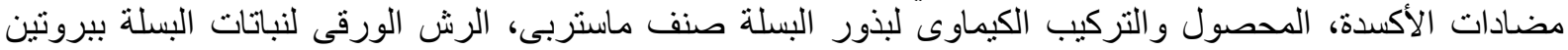

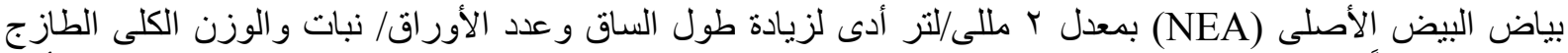

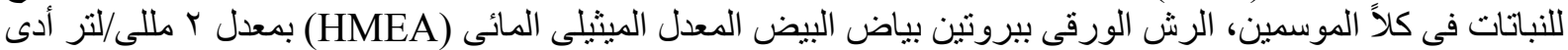

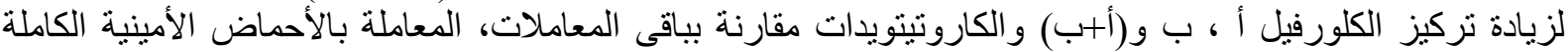

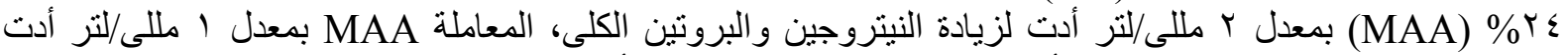

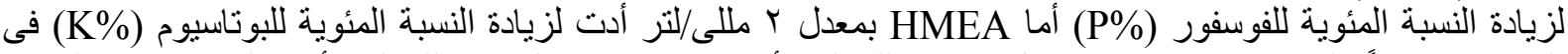

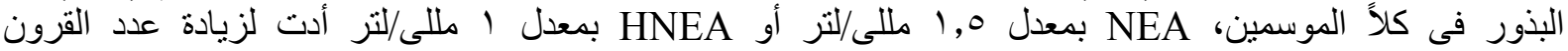

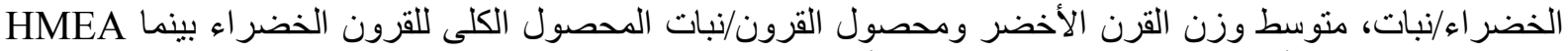

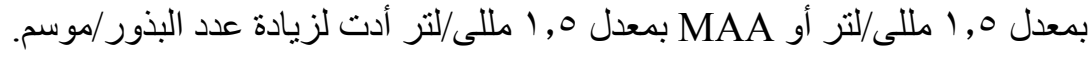

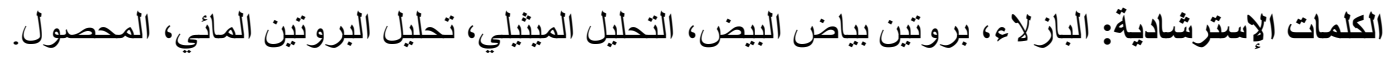

